\title{
Involvement of consumers in the development of evidence based clinical guidelines: practical experiences from the North of England evidence based guideline development programme
}

\author{
A van Wersch, $M$ Eccles
}

\begin{abstract}
Background-Consumer involvement in clinical guidelines has long been advocated although there are few empirical accounts of attempts to do so. It is therefore not surprising that there is a lack of clarity about how and when to involve consumers and what to expect from them within the process of guideline development.

Methods-The North of England evidence based guideline development programme has used four different methods of consumer involvement.

Results-When individual patients were included in a guideline development group they contributed infrequently and had problems with the use of technical language. Although they contributed most in discussions of patient education, their contributions were not subsequently acted on. In a "one off" meeting with a group of patients there were again reported problems with medical terminology and the group were most interested in sections on patient education and self management. However, their understanding of the use of scientific evidence in order to contribute to a more cost effective health care remained unclear. In a workshop it was possible to explain the technical elements of guideline development to patients who could then engage with such a process and make relevant suggestions as a consequence. However, this was relatively resource intensive. A patient advocate within a guideline development group felt confidence to speak, was used to having discussions with health professionals, and was familiar with the medical terminology.
\end{abstract}

Conclusions-Consumers should be involved in all stages of guideline development. While this is possible, it is not straightforward. There is no one right way to accomplish this and there is a clear need for further work on how best to achieve it.

(Quality in Health Care 2001;10:10-16)

Keywords: patient involvement; patient education; guideline development groups

Over the last decade clinical practice guidelines have become an increasingly familiar part of clinical care. Defined as "systematically developed statements to assist both practitioner and
Key messages

- Consumers should be involved in all the stages of guideline development to ensure their views are heard.

- Consumers (like all guideline development group members) need support to be able to understand the detail of the science behind the issues they will hear discussed and to be able to contribute to discussion.

- Having explored four different methods of involving consumers, none was ideal and, even if optimised, each alone would be likely to remain limited.

- Given the greater degree of discussion within a "one off" group of patients than by the sole patients within the guideline groups, it seems reasonable to at least offer consumers within guideline groups the option of being one of a pair.

- It is feasible to support consumers to understand the technical elements of guideline development.

Implications of this work for quality improvement

Consumers should be involved in all the stages of guideline development to ensure their views are heard, but experience on how best to do this is limited. Future guideline development groups should consider (and report on) using more than a single method of consumer involvement, such as involving consumers both within guideline development groups and other structures (focus groups, consumer surveys) outside guideline development groups but feeding into them.

patient decisions in specific circumstances", they are viewed as useful tools for making care more consistent and efficient and for closing the gap between what clinicians do and what scientific evidence supports. ${ }^{2}$ The broad interest in clinical guidelines is international ${ }^{34}$ and has its origin in issues that most healthcare systems face: rising healthcare costs; variations in service delivery with the presumption that at least some of this variation stems from inappropriate care; and the intrinsic desire of healthcare professionals to offer, and patients to receive, the best care possible. Within the UK there is ongoing interest in the development of guidelines ${ }^{5}$ and a fast developing 
clinical effectiveness agenda ${ }^{67}$ within which guidelines figure prominently.

During the same 10 year period the methods of developing guidelines have steadily improved. Overall, methods have moved from those based on unstructured (or even no) review of the evidence, poorly constructed development groups, and solely consensus methods to methods that take explicit account of systematically identified evidence, use multidisciplinary development groups and more structured methods of gathering opinion. However, within this overall improvement there are some areas that remain less well developed and described.

Consumer involvement in clinical guidelines has long been advocated ${ }^{1}$ but there is a realistic perception that attempts at involvement have been driven by political correctness and professional perspectives. ${ }^{8}$ It is striking that, given the volume written about clinical guidelines, there are few empirical accounts of attempts to involve consumers ${ }^{9}$ and suggested frameworks for consumer involvement in guidelines, while useful, appear to have come out of no, or limited, practical experience. ${ }^{8}{ }^{10} \mathrm{It}$ is therefore not surprising that there is a lack of clarity about how and when to involve consumers and what to expect from them within the process of guideline development. ${ }^{11}$

There are good reasons for taking consumer involvement seriously. Firstly, consumers will have a different knowledge, understanding, and experience of the diagnosis and management of the illness process from healthcare professionals. These personal viewpoints and experiences, at an individual or group level, need to be considered when describing the processes of clinical care. A better understanding of the value of the consumer's view is therefore necessary in order to apply these to the guideline development (and implementation) process in the most efficient way.

Within the North of England evidence based guideline development programme 10 evidence based guidelines have been developed and their methods of development have been described. ${ }^{12-15}$ The period of development of these guidelines spanned 6 years over which time there was an evolving experience of involving consumers. Four different methods of consumer involvement were used with some being more successful than others. Three of these were conducted directly within the process of developing guidelines and fed into the guideline recommendations. The other one was conducted as an attempt to explore further methods of involving consumers to inform future guideline development. This paper describes and discusses our experiences with these different methods as a series of case studies and, from this basis, suggests how these methods could be developed.

\section{Methods of involving consumers in guideline development}

The four methods, which were explored in consecutive order, were:

- incorporating individual patients in guideline development groups;
- a "one off" meeting with patients;

- a series of workshops with patients;

- incorporating a consumer advocate in guideline development groups.

The first, second, and fourth were conducted within the development of the guidelines. A range of methods was used including direct observation, individual interviews, and analysis of audio transcripts.

INCORPORATING AN INDIVIDUAL PATIENT IN A GUIDELINE DEVELOPMENT GROUP

For the development of guidelines for two chronic conditions (asthma ${ }^{16}{ }^{17}$ and angina ${ }^{18}{ }^{19}$ ), patients were invited to be members of multidisciplinary guideline development groups. Having failed to identify patients through the Community Health Council, both patients were identified through the secondary care clinicians within the guideline groups. They were invited as individuals who were felt to have both the time and interest to participate. This was our first attempt to involve consumers and, although the two patients were integrated socially into the group, there was neither explicit expectation from their involvement nor any briefing or formal support for them.

\section{Community Health Councils}

Community Health Councils were set up as local independent bodies in 1974 to represent the interests of the public in the National Health Service. They are statutory bodies and have rights to information, to be consulted, and to meet with Health Authorities and National Health Service Trusts.

In addition to the patient, each multidisciplinary group involved primary care, secondary care and public health doctors, primary care nurses, and technical and administrative support staff. The process of guideline development and the methodological issues raised have been previously described. ${ }^{12}{ }^{13}$ The eight meetings of each group were audiotaped and transcribed. In order to analyse the contributions of the patients, the transcripts were content analysed. The summary findings of the two groups are presented together (box 1).

On the whole, the contribution of these patients towards the development of the asthma and angina guidelines was small in comparison with the contributions and communications of the other group members. The most vocal members of the groups were the secondary care physicians who were presenting summaries of the evidence to the other group members. There was a range of rate of contribution from the other (clinical) group members. Although some spoke infrequently, their contributions were usually acted upon. However, the female patient in the asthma group hardly spoke, talking on average only once in a meeting and used, at the most, three sentences each time she spoke. The male patient in the angina group spoke more, on average four 
times in a meeting, and used at the most 12 sentences. These differences might relate to sex or to personality but could also be due to a difference in role perception. The patient in the asthma group contributed to discussions solely by giving illustrations of her own experiences. However, the patient in the angina group, who was also a member of a patient support group, might have seen his role more as a mediator between his patient group and the guideline development group. Besides giving examples as the asthma patient did, he also tried to link the guideline process to the content of the patient's charter. Furthermore, he wanted to develop a flow chart which, it became increasingly apparent, the rest of the group did not want and did not subsequently act upon.

Both patients indicated at several points that they were not familiar with the medical language spoken and asked for clarification of terms and abbreviations. Furthermore, they both contributed most within the discussions on patient education. For the other sections on drugs their main contribution was to give personal examples and this was mostly when specifically asked for their experiences or opinions by the chairperson or other members of the group. Otherwise, they were "participating observers of technical language to which they could hardly offer any input". ${ }^{13}$

- Patients contributed infrequently to the discussions

- Patients had problems with the use of technical language

- They contributed most in discussions of patient education

- Their contributions were not subsequently acted upon

Box 1 Incorporating an individual patient in a guideline development group

“ONE OFF" MEETING WITH PATIENTS

The second method of involving patients in guideline development was discussing an advanced draft version of guidelines with a group of patients at a single evening meeting. The guidelines were an updated version of the previously developed asthma guidelines. ${ }^{20} \mathrm{~Pa}-$ tients were invited to attend via a local group of the National Asthma Campaign. Those who were interested in participating in the meeting were sent a copy of the guidelines and were asked to read these for a forum discussion with the developers of the guidelines. At the meeting the aims of the evening were stated as: (1) to provide the background and rationale of the guidelines; (2) to discuss the necessity of the guidelines and the principle of evidence based information; (3) to explain the meaning of the strengths of the recommended treatments; and (4) to discuss patient input for future guideline development. The discussions were tape recorded, the text transcribed, and the content of the transcripts analysed. The main points will be summarised here (box 2).

The patients constructed the rationale of the guidelines, in the sense of uniform diagnosis and treatment, as positive because of their own
- Patients reported problems with medical terminology and jargon

- They were most interested in sections on patient education and self-management

- Their understanding of the use of scientific evidence in order to contribute to a more cost effective health care remained unclear

Box 2 "One off” meeting with patients

experiences of geographical and professional variation in asthma care. Several patients offered the view that patients with asthma in other parts of the UK received different drugs and advice from them. Furthermore, patients were aware that, in some places, asthma nurses were the main carers while, in other places, doctors were in charge of caring for the patients. Some thought that asthma nurses had more knowledge and understanding of asthma than GPs or consultants because "they have had the training".

Patients were often critical about the treatment they had received for their asthma. Some expressed a lack of confidence in clinicians, and there were several examples of patients having received conflicting information from different clinicians. They also felt they were not always listened to, which led to examples of inappropriate clinical decisions. Furthermore, patients complained about the lack of adequate information: "people go in and out of the hospital without being told what is happening"; "there is no one to ask questions from".

Patients also identified problems with the use of medical terminology and jargon by clinicians, saying that this did not help in understanding what was going on. As specific examples, patients highlighted the word "steroids" and the use of differing terms for drug delivery devices. They felt that when doctors used the term "steroids" as the prescribed drugs for asthma they seemed to forget the negative connotations this might have for patients for whom steroids are seen as forbidden drugs taken by top athletes. The other aspect mentioned by patients was the naming of drug delivery devices. Patients reported thinking about the different inhalers by their colour. They described knowing exactly when to use the blue inhaler, and when to get the brown, orange, or green one. Furthermore, they were more used to the brand names than the generic names of the various drugs. Healthcare professionals, on the other hand, were felt to use the "chemical" names, as reflected in the guidelines. This difference of terminology seemed to lead to unnecessary misunderstandings in the care process.

The sections of the guidelines that attracted patients most were those on patient education and self-management. However, personal experience rather than the available evidence influenced the views they expressed. The use of peak flow meters and flow charts of readings were examples of where they suggested patterns of use that did not match with the content of the guideline. However, they did identify 
that they would like to have received information on asthma in layman's terms and in a more simplified form. Interestingly, some patients had brought information leaflets with them to the meeting and pointed out the contradictions between them. However, most of these leaflets were written by pharmaceutical companies promoting their own drugs. Efforts to explain to the patients the role of these companies in health care were not very successful.

Overall, the patients were interested in the content of the meeting and their response to the idea of guidelines was positive: "everything's got its own rules-you need rules to play hockeythese are asthma rules". Patients did show an understanding of the applicability of the guidelines for more uniform diagnosis and treatment; however, the underlying idea of the use of scientific evidence to contribute to a more cost effective health care remained unclear.

WORKSHOPS WITH PATIENTS

The third method was the development of a series of workshops to explore the potential to increase patients' understanding of the meaning of scientific evidence, their ideas of cost effectiveness, and their views on patient information. This method was explored outside the guideline development process as an exercise to see how much of the technical content of the guideline could be explained to consumers. The guideline was the same as that used in the single meeting with patients and the patients were again recruited through the local branch of the National Asthma Campaign. Four workshops were run by a psychologist member of the research team (AvW) with an average attendance of 10 patients. In the first two a role play was carried out. In the last two sessions patient information was explored in more detail and suggestions for a patient version of the asthma guidelines were discussed.

The scenario for the first role play was the organisation of a party. Each patient received a budget of $£ 50$ to do the shopping for a party at home with 10 guests. They were asked to write their shopping list and to prioritise the goods they wished to buy. Each patient then told the others in the group what they had bought and the reasons for their choices.

The first observation was that nobody had exactly the same shopping list. Some prioritised drinks, others food. The reasoning behind their choices differed as well. For example, for the wine, four reasons for their choices were mentioned: alcohol percentage of the wine, price per bottle, habit (choosing the wine one always drinks), and chance (choosing the wine which happens to be on offer). Finally, there was a difference in quality and quantity. Some patients ended up with only half as many groceries as others on the rationale of "the more expensive, the better the quality". This led to a debate on quality and quantity, and how to measure quality. The group was then asked to set up a study to measure differences in quality of bottles of wine costing $£ 10$ and $£ 2.50$. They would ask people to drink both wines without telling them which was which. They would then question them about their preferences in taste, colour, and price. Thus, the group designed a simple trial. Following these role plays, both randomised trials with asthma drugs and the principle of evidence based recommendations were subsequently explained to the patients with no difficulty.

In the second workshop the group was asked to assume the role of the person responsible for purchasing at their local district health authority. With a budget of $£ 500$ they had to purchase inhalers for their asthma patients. Four different companies offered inhalers to them, each claiming their own as the most effective. The inhalers differed in cost $(£ 1, £ 5, £ 15$, and $£ 30$ ), form (aerosol, powder), consequences (taste, side effects), and delivery device (colour, size, design, mechanism). All four inhalers came with an information leaflet claiming the excellence of their product. The group showed an increased awareness of reasons other than cost in their choices, such as the demand for inhalers, patient characteristics, and patient preferences. None of the group members was inclined to just buy the cheapest or the most expensive (thus not following the principle "the more expensive, the better"). Overall, the group thought they could not make a responsible choice before independent (from the pharmaceutical industry) studies had shown which inhalers were better for which group of patients (children, older people, people with or without other ailments).

In the last two workshops information leaflets and brochures on asthma were studied in more detail. At the end of the third workshop agreement was reached that a patient version of the evidence based guidelines would be the most reliable and up to date information for patients. In the last workshop the development of an evidence based guideline for asthma patients was explored and several ideas were suggested by the group. These included translating medical jargon into laymen's terms, the layout of the document (for example, use of colour, pictures and a bigger font), suggestions on the contents (for example, use of "strong evidence" and "weak evidence" instead of the letters A, B, C, and D), inclusion of a peak flow booklet, use of colours and brand names by inhalers and drugs, and explanation of the use of steroids.

This method suggested that it is possible to explain the technical elements of guideline development to patients and that they can engage with such a process and make relevant suggestions as a consequence. However, the process was relatively resource intensive (box 3 ).

INCORPORATING A PATIENT ADVOCATE IN A GUIDELINE DEVELOPMENT GROUP

The final method used was the involvement of a consumer advocate as a member of the group developing a guideline on the secondary preventive treatment of patients who have survived a myocardial infarction. The consumer advocate was the head of a national cardiac patient group and was recruited directly after her involvement in the development of the national service framework on coronary heart 
disease. A single telephone interview was conducted covering the experiences and satisfaction of the patient advocate herself and her involvement in the development group.

- It was possible to explain the technical elements of guideline development to patients

- Patients could engage with such a process and make relevant suggestions as a consequence

- The process was relatively resource intensive

Box 3 Workshops with patients

She saw her role as liaising between (her) patient group and the guideline group. Because of her experiences working for the cardiac patient association, she reported not feeling overpowered by the other (healthcare professional) group members. She felt she had enough confidence to speak up, mainly because of her perceived equivalence of her participatory role in the guideline group with the tasks of her job. She had previous similar experiences, was used to having discussions with health professionals, was familiar with the medical terminology and, if there was anything she did not understand, she had access to several sources to resolve this.

She felt the group worked well and that her input and output were well balanced. She felt that she got across to the other group members the points she wanted to share. In addition, she felt that she had learned a lot from the others in the group.

Based on her experiences, she felt that the ideal consumer representative within a guideline group should have a number of characteristics. She suggested that they should not themselves be a patient, should have a broad knowledge of the physical condition and patients' experiences of coping with the illness, should have involvement in patients' feelings, problems and situations, have an understanding of patients' needs, and training in counselling or communication skills. She also felt that having personal experience with a patient was an advantage (box 4 ).

- The advocate had previous similar experiences

- She felt confidence to speak within the group

- She was used to having discussions with health professionals

- She was familiar with the medical terminology

Box 4 Incorporating a patient advocate in a guideline development group

\section{Discussion}

Four different methods of patient involvement in evidence based clinical guidelines have been described, three of which were used in the process of developing guidelines and one was conducted to explore the potential of the method for future use. According to Williamson $^{21}$ three types of patient representatives within working committees can be distinguished, depending on the contributions and skills each can be expected to bring: (1) fellow patients, who would mainly present their own views; (2) member of a patient group, who would present the group's views; and (3) patient advocates, who would present knowledge of patient views. In this paper we have described our experiences with all three types.

When individual patients were incorporated into the guideline development groups their contribution was small. According to group dynamics theory, ${ }^{22}$ this could have been anticipated since one of the hypotheses is that "the high status person both initiates and receives more communication than the low status person (p 286)". It now seems self-evident that patient involvement in a guideline group will not work if the patient does not receive enough information on the aims of, and their role in, the group, is not briefed properly, and does not feel comfortable in the group. Were this method to be attempted again, as it surely will, it would be important to address these issues. Given the greater degree of discussion within the "one off" group of patients than by the sole patients within the guideline groups, it seems reasonable to at least offer consumers within guideline groups the option of being one of a pair. This would not only provide more tangible support, but also lessen the risk of only hearing from a "lone" or "token" consumer.

The involvement of patients through the single meeting and the workshops produced more interaction and discussion. In the "one off" meeting patients were able to raise issues of concern from their reading of the guideline and to identify a number of areas where the guideline could be altered to make it more accessible to non-clinicians. One of the main stumbling blocks with this method was that it was not possible to discuss meaningfully any of the "scientific" content of the guideline and therefore patients could only talk from the basis of their personal experience. However, from the workshops it became apparent that patients could understand the rationale of both scientific evidence and cost effectiveness in health care when allowed to work through day to day examples. However, to achieve this was relatively resource intensive, taking four evening meetings. Such resources may well be beyond the scope of individual guideline development groups but could be resourced by broader guideline development programmes.

Our experiences with a patient advocate showed this to be a feasible method of getting consumer involvement. Personal involvement with an illness is different for a patient than for a patient advocate. While discussing patients' matters, health professionals will objectify and generalise patients' experiences. They will not do this to deny the personal experience of the patient, but to understand the commonality of the problem. While this could be distressing for the patients themselves, it should not be for the patient advocate. Additionally, the presence of a patient might inhibit the health professionals 
in their open communications, which might be less with a patient advocate being present. A patient advocate, rather than a patient or member of a patient support group, also goes some way towards addressing the issue of differences in status within multidisciplinary working groups.

Having involved consumers within the guideline development process, their contributions did not necessarily alter the content of the guidelines. There are a number of possible reasons for this. Firstly, the scope of the guidelines had been defined in fairly narrow medical terms and at a stage when consumers were not involved. Secondly, the process of guideline development - with its focus on validity and underlying evidence-deals less comfortably with "non-evidence based" views and preferences. The Institute of Medicine ${ }^{1}$ suggested that consumer views should influence recommendations where there is no, or equivocal, evidence. This is borne out by our experiences in that patients were content with being guided by evidence. In areas where there was no evidence (such as how patients should be treated interpersonally), all members of guideline groups supported general statements about ensuring good clinician/patient communication.

These experiences highlight the issues of how consumers should be involved in all the stages of guideline development and what is expected of them. Although each of the four methods had advantages (and disadvantages), none was ideal and, even if optimised, each alone would be likely to remain limited. Consumers need to sit as members of guideline groups to ensure their views are heard. To avoid hearing only a single view (where it is likely there is a range), broader views can be gathered from outside the groups. This could be addressed by using more than a single method of consumer involvement, an approach that would fit with the highest of Bastian's "levels of involvement". 8 This would mean involving consumers both within guideline development groups and in other structures (focus groups, consumer surveys) outside guideline development groups but feeding into them. Guideline development groups are now starting to use such multiple methods. The guideline development group for the stroke guideline produced by the Royal College of Physicians contained three patient organisation representatives but also used focus groups to explore patient and carer views. ${ }^{23}$

It is less clear how much support needs to be provided to help consumers (or other group members) to understand the detail of the science behind the issues they will hear discussed. To a greater or lesser extent, most members of a guideline development group will have limitations in their ability to understand the detail of systematic review or health economics, and the guideline development process allows that these specialised skills will be provided by specialists within the groups. Appropriate support and training therefore needs to be supplied to all members of guideline development groups to allow them to

\section{National Institute for Clinical Excellence (NICE)}

The National Institute for Clinical Excellence (NICE) was set up as a Special Health Authority for England and Wales on 1 April 1999. It is part of the National Health Service and its role is to provide patients, health professionals, and the public with authoritative, robust and reliable guidance on current "best practice". Guidance covers both individual health technologies (including medicines, medical devices, diagnostic techniques, and procedures) and the clinical management of specific conditions.

fulfil their role. It is reasonable to suppose that some issues (hierarchies within groups, medical terminology) will be particularly heightened for consumers. Having achieved this, in the absence of any specific shared role for group members (such as sharing the reviewing task), the role of a consumer then becomes the same as that of any other group member-to comment on the process of care and the issues around this in the light of the available summarised evidence. From the content of the meeting and the workshops it is likely that this will produce comment on the content and also on the wording of recommendations and the presentation of the guideline, both in general and particularly if a specific patient version is to be produced.

The National Institute for Clinical Excellence is actively promoting consumer involvement within guidelines developed within its programme (M Kelson, personal communication). There is a growing body of guidance on supporting user representatives to promote more effective involvement in other areas of NHS activity. ${ }^{24}{ }^{25}$ This will inform the methods of involving consumers within guideline development. Our experiences to date and that of others ${ }^{24}$ suggests that it is possible to get meaningful consumer involvement into guideline development but that this is not straightforward. There is no one right way to accomplish this and there is a clear need for further work on how best to achieve it.

We are grateful to all of the consumers who have been involved in the guideline development processes described; the National Asthma Campaign for their support in setting up and running the "one off" meeting and the series of workshops; the Department of Health for England and Wales for funding the guideline development; and Nikki Rousseau for her help in running the "one off" meeting.

1 Field MJ, Lohr KN, eds. Guidelines for clinical practice: from development to use. Washington DC: National Academy Press, 1992

2 Woolf SH, Grol R, Hutchinson A, et al. Clinical guidelines: the potential benefits, limitations and harms of clinical guidelines. BMF 1999;318:527-30.

3 Grol R, Eccles M, Maisonneuve H, et al. Developing clinical practice guidelines: the European experience. Dis Manage Health Outcomes 1998;4:255-66.

4 Woolf S, Grol R, Hutchinson A, et al. An international overview. In: Eccles M, Grimshaw J, eds. Clinical guidelines: from conception to use. Oxford: Radcliffe Medical Press, 2000 : conception $31-48$.

5 NHS Executive. Clinical guidelines: using clinical guidelines to improve patient care within the NHS. London: HMSO, 1996. improve patient care within the NHS. London: HMSO, 1996.
Department of Health. The new NHS: modern, dependable. Department of Health. The new NHS:
London: Department of Health, 1997.

7 NHS Executive. A first class service: quality in the new NHS. NHS Executive. A first class
London: HMSO, 1998. 
8 Bastian H. Raising the standard: practice guidelines and castian $H$. Raising the standard: practice guidelines and consumer

9 Carver AD, Entwistle V. Patient involvement in SIGN guideline development groups. Edinburgh: Scottish Association of Health Councils, 1999

10 Duff L, Kelson M, Marriott S, et al. Clinical guidelines: involving patients and users of services. F Clin Effect 1996 1:104-12.

11 Van Wersch A, Eccles M. Patient involvement in evidencebased health in relation to clinical guidelines. In: Gabbay $\mathrm{M}$, ed. The evidence-based primary care handbook. London: Royal Society of Medicine, 1999: 91-103.

12 Eccles MP, Clapp Z, Grimshaw JM, et al. North of England evidence based guidelines development project: methods of guideline development. BMҰ 1996;312:760-1.

13 Eccles MP, Clapp Z, Grimshaw J, et al. Developing valid guidelines: methodological and procedural issues from the North of England evidence based guideline development project. Ouality in Health Care 1996;5:44-50.

14 project. Quality in Health Care 1996;5:44-50. evidence based guideline development project: methods of developing guidelines for efficient drug use in primary care. BMF 1998;316:1232-5.

15 Eccles M, Mason J, Freemantle N. Developing valid cost effectiveness guidelines: a methodological report from the North of England evidence based guideline development project. Quality in Health Care 2000;9:127-32.

16 North of England Evidence Based Guideline Development Project. Evidence based clinical practice guideline: the primary care management of asthma in adults. Report 75. Centre for Health Services Research, Newcastle upon Tyne, 1996.
17 North of England Asthma Guideline Development Group. North of England evidence based guidelines development project: summary version of evidence based guideline for the primary care management of asthma in adults. $B M F$ 1996;312:762-6.

18 North of England Evidence Based Guideline Development Project. Evidence based clinical practice guideline: the primary care management of stable angina. Report 74. Centre for Health Services Research, Newcastle upon Tyne, 1996.

19 North of England Stable Angina Guidelines Development Group. Evidence based guideline for the primary care management of stable angina: summary version. BMF 1996;312:827-32.

20 North of England Evidence Based Guideline Development Project. The primary care management of asthma in adults. Report 97. Centre for Health Services Research, Newcastle upon Tyne, 1999.

21 Williamson C. The rise of doctor-patient working groups. BMf 1998;317:1374-7.

22 Shaw ME. Group dynamics. The psychology of small group behaviour. New Delhi: Tata McGraw-Hill Publishing, 1977.

23 The Intercollegiate Working Party for Stroke. National clinical guidelines for stroke. London: Royal College of Physicians, 2000

24 Kelson M. User involvement: a guide to developing effective user involvement strategies in the NHS. London: College of Health, 1997.

25 Consumers in NHS Research Support Unit. Involving consumers in research and development in the NHS: briefing notes for researchers. Winchester: Help for Health Trust, 2000 .

\section{Cambridge International Health Leadership Programme \\ TRANSFORMING HEALTH: CREATING VALUE THROUGH CHANGING SYSTEMS}

An intensive 8 day international seminar for health sector leaders

\section{8-25 April 2001 \\ Cambridge, UK}

The Cambridge International Health Leadership Programme has been established to provide an annual forum in which senior health sector leaders from around the world address the issues of health sector reform in the face of rapid change and development. The seminar provides access to essential information and unparalleled management and policy expertise. In consultation with leading international figures in the field, participants in this, the fifth seminar in the series, will be able to identify ways to improve the performance of their country's health sector for the 21 st century.

Through a combination of lectures, workshops, case analyses, and discussion groups the Programme will strengthen and enrich the ability of participants to manage complexities and improve performance. Group work on selected topics is integrated into the programme, constructed around participants' own issues, giving delegates the opportunity to learn from each other as well as with the faculty. They will learn the latest approaches to quality improvement and will have the opportunity to develop their own plans and approach to managing and leading change.

Participation in the seminar will link delegates into an influential network involving both their peers, faculty, and the agencies supporting and driving health sector reform.

The seminar is organised on behalf of the Judge Institute of Management Studies by the University of Cambridge Programme for Industry.

\section{Location}

Sessions will be held at the Judge Institute of Management Studies in its stunning new building in the centre of Cambridge. The Judge Institute is the University of Cambridge's business school.

\section{Programme Fee: $£ 4900$ (no VAT)}

The fee includes all documentation, seminar notes, full accommodation (nights of Wednesday 18 April to Tuesday 24 April 2001), meals and other refreshments throughout the Seminar. The fee does not include travel expenses.

A limited number of bursaries are available for the event.

\section{Nomination enquiries/further information}

Please contact:

Francesca Barraud, Programme Manager, University of Cambridge Programme for Industry, 1 Trumpington Street, Cambridge CB2 1QA, UK

Tel: +44 (0)1223 342100. Fax: +44(0)1223 301122

email: francesca.barraud@cpi.cam.ac.uk 